\title{
Differential Effects of Lactobacillus casei Strain Shirota on Patients With Constipation Regarding Stool Consistency in China
}

\author{
Shanbin Chen, ${ }^{1}$ Yangwenshan Ou, ${ }^{2}$ Liang Zhao, ${ }^{1,3}$ Yang Li, ${ }^{4}$ Zhenxing Qiao, ${ }^{4}$ Yanling Hao, ${ }^{1,2,5}$ and Fazheng Ren ${ }^{1,3 *}$ \\ ${ }^{\prime}$ Beijing Advanced Innovation Center for Food Nutrition and Human Health, College of Food Science and Nutritional Engineering; China \\ Agricultural University, Beijing, China; ${ }^{2}$ Beijing Laboratory for Food Quality and Safety, Beijing; China; ${ }^{3}$ Key Laboratory of Functional Dairy, \\ College of Food Science and Nutritional Engineering, Beijing, China; ${ }^{4}$ Science and Public Relations Office, Yakult (China) Corporation, Shanghai, \\ China; and ${ }^{5}$ Beijing Key Laboratory of Detection and Control of Spoilage Organisms and Pesticide Residues in Agricultural Products, Beijing \\ University of Agriculture, Beijing, China
}

\begin{abstract}
Background/Aims
Probiotics are expected to confer benefits on patients with constipation, but how probiotics act on constipated patients with variable stool consistencies remains unclear. We investigated the effect of Lactobacillus casei strain Shirota (LCS) on constipation-related symptoms, especially stool consistency, of constipated patients.

\section{Methods}

Constipated patients meeting the Rome III criteria were divided into 3 groups according to the Bristol Stool Form Scale (BSFS): hard (hard stool [HS], BSFS $<3$ ), normal (normal stool [NS], $3 \leq$ BSFS $\leq 4$ ), and soft (soft stool [SS], $4<$ BSFS $\leq 5$ ) stools. Subjects in each group consumed a probiotic beverage containing $10^{10}$ colony-forming units of LCS daily for 28 days.

\section{Results}

LCS intervention significantly alleviated constipation-related symptoms and increased defecation frequency in all subjects. Four weeks of LCS supplementation softened the hard stools in HS, hardened the soft stools in SS, and did not alter the ideal stool consistency in NS. The short-chain fatty acid (SCFA) concentrations were highest in SS, followed by NS and HS. LCS intervention increased the stool SCFA levels in HS but reduced or did not alter the levels in NS and SS. LCS intervention increased the Pseudobutyrivibrio and Roseburia abundances in HS and decreased the Pseudobutyrivibrio abundance in SS.
\end{abstract}

\section{Conclusions}

LCS supplementation improved the constipation-related symptoms in constipated subjects. Differences in baseline stool consistency could result in different anti-constipation effects of LCS intervention. LCS balanced the stool consistency-softened the HS and hardened the SS. These effects could be associated with modulation of the gut microbiota and SCFA production.

(J Neurogastroenterol Motil 2019;25:148-158)

Key Words

Constipation; Fatty acids, volatile; Gastrointestinal microbiome; Lactobacillus casei

Received: May 14, 2018 Revised: July 11, 2018 Accepted: July 31, 2018

@) This is an Open Access article distributed under the terms of the Creative Commons Attribution Non-Commercial License (http://creativecommons. org/licenses/by-nc/4.0) which permits unrestricted non-commercial use, distribution, and reproduction in any medium, provided the original work is properly cited.

${ }^{*}$ Correspondence: Fazheng Ren, PhD

Beijing Advanced Innovation Center for Food Nutrition and Human Health, College of Food Science and Nutritional Engineering, China Agricultural University, Beijing, 100083, China

Tel: +86-010-6273-8589, Fax: +86-010-6273-6344, E-mail: renfazheng@cau.edu.cn

Shanbin Chen and Yangwenshan Ou contributed equally to this study. 
tencies and examined the microbiota underlying this difference.

\section{Introduction}

Constipation is common but bothersome, affecting approximately $8.2 \%$ of the Chinese population. ${ }^{1}$ Constipation, at its worst, can reduce work productivity, interfere with daily life activities, impair the quality of life, and lead to high costs of health maintenance. $^{2,3}$

The Rome III criteria, a widely used diagnostic tool for assessing functional constipation, was created based on a diverse range of subjective or measurable symptoms associated with bowel movement frequency, stool consistency (stool hardness), and easiness of defecation. ${ }^{4}$ Patients diagnosed as constipated based on the Rome III criteria had diverse symptoms, including straining during defecation, hard stools, the urge to defecate with an inability to evacuate, infrequent stools, abdominal discomfort, a sensation of incomplete evacuation, and excessive defecation time. ${ }^{5}$ Of all the symptoms, stool consistency is one of the most critical diagnostic indicators. However, stool consistency is not the only factor considered by the Rome III criteria. ${ }^{6}$ It is possible to diagnose a patient with soft stool as being constipated. It was also reported that soft stools are often a complaint of patients with functional constipation and those who defecate three or more times per week. ${ }^{7}$

Dysbiosis of the gut microbiota has been hypothesized to contribute to the onset and clinical manifestation of constipation. Probiotics are widely used to modify the gut microbiota. ${ }^{8}$ Systematic reviews of randomized controlled trials have suggested the beneficial effects of probiotics on constipation, but the outcomes varied greatly. ${ }^{9,10}$ For example, Bifidobacterium lactis lactis was reported to improve stool consistency, but contradictory results were also observed. ${ }^{11,12}$ Lactobacillus casei strain Shirota $(\mathrm{LcS})$ is also thought to be a promising strain for the alleviation of constipation, but the outcomes of studies on the effects of this strain have not been entirely consistent. ${ }^{10}$ Stool consistency might account for this discrepancy, as it was reported that $\mathrm{LcS}$ administration reduced hard stool consistency in constipated patients but had no effect on constipated patients with normal stool consistency. ${ }^{13-15}$ Thus, we investigated the different effects of LcS on constipation in patients with distinct stool consis-

\section{Materials and Methods}

\section{Study Subjects}

The study protocol was reviewed and approved by the ethics committee of the People's Liberation Army Beijing Military General Hospital (research project identification No. 2015/105). All subjects provided signed informed consent. Subjects aged 18-45 were recruited from Beijing, China, and screened according to the Rome III criteria for functional constipation. The exclusion criteria for this crowd experiment were as follows: serious diseases, organic or neurological lesions, systemic antibiotic or antimycotic use, antidiarrheal or laxative use (30 days prior the study), milk allergy, and participation in other similar studies (2 months prior to the study).

\section{Study Design}

This open trial consisted of a 2-week baseline period, a 4-week intake period, and a 2-week follow-up period in succession (Fig. 1). During the intake period, after their daily lunch, the subjects consumed 1 bottle of the commercial beverage Yakult (an LcS-fermented dairy beverage; $100 \mathrm{~mL}$, containing $1 \times 10^{10}$ living LcS cells; sponsored by Yakult (China) Corp, Shanghai, China). Subjects were required to maintain their eating habits throughout the study. Other fermented dairy products, laxatives, antibiotics or medications that might interfere with study outcomes were not allowed.

Subjects were required to rate the severity of constipationrelated symptoms as described by Sakai. ${ }^{13}$ Painful effort, feeling of incomplete evacuation and straining during defecation were assessed on a 4-point scale (1, none; 2, little; 3, some; and 4, a lot). The number of minutes spent in the lavatory per attempt was also assessed: less than 5 minutes was scored as 1, no less than 5 and less than 10 as 2, no less than 10 and less than 20 as 3, more than 20 as 4 . Unsuccessful defecation attempts and abdominal discomfort were recorded in the diary on a daily basis (1, never; 2 , rarely; 3 , sometimes; and 4, always).

Stool consistency assessed by the Bristol Stool Form Scale

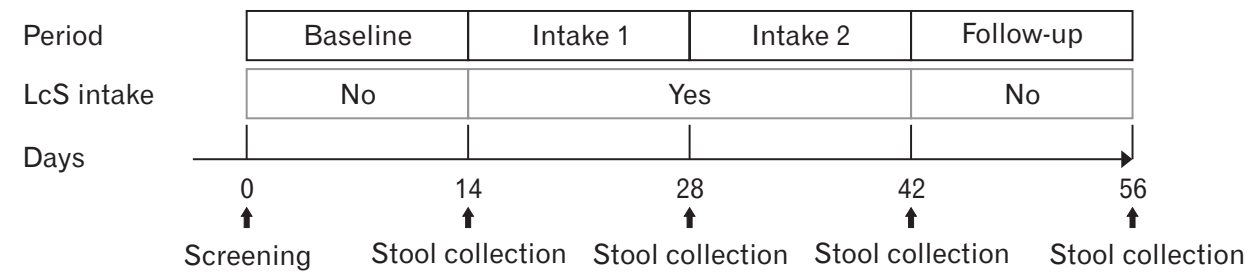

Figure 1. Study design. The subjects consumed only Lactobacillus casei strain Shirota ( $\mathrm{LcS}$ )-fermented dairy beverage during the intake period. Intake 1 , the first 2-week LcS intake period; intake 2 , the second 2-week LcS intake period; follow-up, 2-week following LcS intake. 
(BSFS) and defecation frequency were recorded in the daily diary. ${ }^{16}$ The BSFS classified stool into 3 categories according to score: 1-2, hard stool (HS); 3-4, normal stool (NS); and 4-5, soft stool (SS). Subjects received adequate training before using the BSFS. Then, the subjects were assigned into these 3 categories on the basis of the BSFS. ${ }^{17}$ As shown in Figure 1, stool samples were collected on days 14 (baseline visit), 28 (intake 1 visit), 42 (intake 2 visit), and 56 (follow-up visit) according to the study schedule. Stool samples were immediately transported to the laboratory in cold containers $(<$ $10^{\circ} \mathrm{C}$ ) and stored at $-80^{\circ} \mathrm{C}$ until analysis.

\section{Measurement of Short-chain Fatty Acid Levels}

Short-chain fatty acids (SCFAs) were extracted from the stool samples as previously described, with slight modifications. ${ }^{18}$ Briefly, $0.1 \mathrm{~g}$ of the stool samples were homogenized by a Mini Beadbeater-16 (BioSpec Products, Inc, Bartlesville, OK, USA) in $1 \mathrm{~mL}$ of diethyl ether containing $5 \mathrm{mmol} / \mathrm{L}$ heptatonic acid (internal standard) and $0.05 \mathrm{~mol} / \mathrm{L} \mathrm{HCl}$ (which acidified the mixture to form free SCFAs). Samples were centrifuged at $12000 \times \mathrm{g}$ for 1 minute at $4^{\circ} \mathrm{C}$. The supernatant was extracted and used for SCFA detection.

SCFA levels were measured by gas chromatography (GC). The GC system consisted of a GC 7890A equipped with a flame ionization detector (Agilent Technologies, Inc, Santa Clara, CA, USA) and an HP-FFAP chromatographic column $(25 \mathrm{~m} \times 0.32$ mm i.d., $1 \mu \mathrm{m}$ ) (Agilent Technologies, Inc). The oven temperature was set as follows: maintained at $50^{\circ} \mathrm{C}$ for 3 minutes, increased at $5^{\circ} \mathrm{C} / \mathrm{min}$ to $140^{\circ} \mathrm{C}$ for 1 minute, and increased at $30^{\circ} \mathrm{C} / \mathrm{min}$ to $240^{\circ} \mathrm{C}$ for 3 minutes. The injector temperature was $27^{\circ} \mathrm{C}$, and the injection type was splitless. Nitrogen was used as the carrier gas, and $1 \mu \mathrm{L}$ of the supernatant was injected for GC measurement.

\section{Statistics}

Data are reported as the mean $\pm \mathrm{SD}$ unless otherwise indicated. Statistical analysis was performed with SPSS Version 21 (IBM Corp, Armonk, NY, USA). The Wilcoxon signed-rank test was applied to compare data between periods. The Wilcoxon ranksum test was applied to compare groups. Spearman correlation was used to evaluate BSFS scores and SCFA levels in RStudio (version 0.99.902). Correlations were considered significant when $P<0.05$ (adjusted for a false discovery rate).

\section{Illumina MiSeq Sequencing of the V3-V4 Regions of the Stool Bacterial 16S Ribosomal RNA Genes}

DNA was extracted from the stool samples using the phenolchloroform method as described by Via and Falkinham. ${ }^{19}$ The V3-
V4 regions of the bacterial 16S ribosomal RNA genes were amplified by polymerase chain reaction (PCR) $\left(95^{\circ} \mathrm{C}\right.$ for 3 minutes, followed by 28 cycles of $95^{\circ} \mathrm{C}$ for 30 seconds, $55^{\circ} \mathrm{C}$ for 30 secconds, and $72^{\circ} \mathrm{C}$ for 45 seconds and a final extension at $72^{\circ} \mathrm{C}$ for 10 minutes) using the primers 338F (5'-barcode-ACTCCTACGGGAGGCAGCAG-3') and 806R (5'-GGACTACHVGGGTWTCTAAT-3'), where the barcode was an 8-base sequence that was unique to each sample. PCRs were performed in triplicate $20-\mu \mathrm{L}$ mixtures containing $4 \mu \mathrm{L}$ of $5 \times$ FastPfu buffer, $2 \mu \mathrm{L}$ of $2.5 \mathrm{mmol} /$ L dNTPs, $0.8 \mu \mathrm{L}$ of each primer $(5 \mu \mathrm{mol} / \mathrm{L}), 0.4 \mu \mathrm{L}$ of FastPfu polymerase, and $10 \mathrm{ng}$ of template DNA.

Amplicons were extracted from 2\% agarose gels and purified using the AxyPrep DNA Gel Extraction Kit (Axygen Biosciences, Inc, Union City, CA, USA) according to the manufacturer's instructions and quantified with a QuantiFluor-ST (Promega Corp, Sunnyvale, CA, USA). Purified amplicons were pooled at equimolar concentrations and subjected to paired-end sequencing $(2 \times$ 250) on an Illumina MiSeq platform (Illumina, Inc, San Diego, CA, USA) according to standard protocols. The raw reads were deposited into the National Center for Biotechnology Information Sequence Read Archive database.

Raw FASTQ files were demultiplexed and quality-filtered using QIIME (version 1.17) with the following criteria: (1) 300-bp reads were truncated at any site receiving an average quality score $<20$ over a 50-bp sliding window, discarding the truncated reads that were shorter than 50 bp. (2) Reads with incomplete barcode matches, 2-nucleotide mismatches in the primer annealing sites, or ambiguous bases were removed. (3) Only sequences that overlapped by more than $10 \mathrm{bp}$ were assembled according to their overlap sequence, and reads that could not be assembled were discarded.

Operational taxonomic units (OTUs) were clustered with a 97\% similarity cutoff using UPARSE (version 7.1; http://drive5. com/uparse/), and chimeric sequences were identified and removed using UCHIME analyses. Sequences aligned using ClustalW2 were used to construct a neighbour-joining tree with the $\mathrm{R}$ package ape. The tree and OTU abundance were then used to calculate weighted UniFrac distances with the R package GUniFrac. OTUs with proportional abundances of at least $0.1 \%$ in at least 3 samples were retained for downstream analysis. The taxonomy of each $16 \mathrm{~S}$ ribosomal RNA (rRNA) gene sequence was analysed by RDP Classifier (http://rdp.cme.msu.edu/) against the silva (SSU115) $16 \mathrm{~S}$ rRNA database using a confidence threshold of $70 \% .^{20}$

\section{Bioinformatics and Statistical Methods}

Samples were rarefied to a depth of 17764 reads per sample. 
The relative abundances of different genera in each sample were calculated with RStudio. To identify the differences in stool microbial groups among the 3 groups at the baseline period or during LcS supplementation, repeated measures ANOVA was conducted by RStudio to evaluate the relative abundance of the intestinal flora at the genus level. ${ }^{21}$ Principal coordinate analysis (PCoA) based on weighted UniFrac distances of the OTUs was performed to obtain an overview of differences in the gut microbes between groups. Analysis of similarities (ANOSIM) was applied to compare weighted UniFrac distances between and within periods. Weighted UniFrac is a quantitative measure of diversity that can determine changes in the number of sequences from each lineage present as well as changes in the taxa present. ${ }^{22}$

\section{Results}

\section{General Subject Characteristics}

Eighty-two subjects enrolled in this study. There were 18 withdrawals due to continued noncompliance with the requirements of the protocol (did not consume Yakult for 2 consecutive days, did not collect adequate stool samples, or did not submit stool samples on time) and 2 dropouts for personal reasons. A total of 62 constipated subjects completed the study, including 54 females and 8 males. There were 23, 27, and 12 subjects in the HS, NS, and SS groups, respectively. No significant differences were observed among these 3 groups in terms of sex, height, weight, and body mass index (Table 1), but the subjects in the HS group were significantly older than the others.

\section{Effect of Lactobacillus casei Strain Shirota on Constipation-related Symptoms in All the Subjects}

For all the subjects, 28 consecutive days of LcS supplementation significantly alleviated some constipation-related symptoms (including painful effort during defecation, feeling of incomplete evacuation, straining during defecation, minutes spent in the lavatory per attempt and abdominal discomfort) and helped increase the defecation frequency and BSFS score (Table 2).

Table 1. Subject Demographics

\begin{tabular}{|c|c|c|c|c|}
\hline \multirow{2}{*}{ Variable } & \multicolumn{3}{|c|}{ BSFS } & \multirow{2}{*}{$P$-value } \\
\hline & $\operatorname{HS}(\mathrm{n}=23)$ & NS $(n=27)$ & $\mathrm{SS}(\mathrm{n}=12)$ & \\
\hline Sex ( $\%$ of females) & 83 & 85 & 100 & $>0.999^{\mathrm{a}}$ \\
\hline Age (yr) & $27.5(10.8)$ & $24.0(7.0)$ & $23.0(3.8)$ & $0.007^{\mathrm{b}}$ \\
\hline Height (m) & $1.63(0.13)$ & $1.63(0.10)$ & $1.64(0.06)$ & $0.851^{\mathrm{b}}$ \\
\hline Weight (kg) & $57.5(7.8)$ & $55.0(15.0)$ & $52.5(8.3)$ & $0.503^{\mathrm{b}}$ \\
\hline $\mathrm{BMI}\left(\mathrm{kg} / \mathrm{m}^{2}\right)$ & $21.0(2.4)$ & $20.7(3.5)$ & $19.6(1.7)$ & $0.448^{\mathrm{b}}$ \\
\hline
\end{tabular}

BSFS, Bristol Stool Form Scale; HS, hard stool; NS, normal stool; SS, soft stool; BMI, body mass index.

$P$-value was calculated by ${ }^{\mathrm{a}} \mathrm{Chi}$-squared test, ${ }^{\mathrm{b}} \mathrm{Kruskal}$ Wallis test.

Data are presented as median (interquartile range).

Table 2. Effects of Lactobacillus casei strain Shirota on Constipation Symptoms and Defecation Frequency

\begin{tabular}{lcccc}
\hline \multicolumn{1}{c}{ Symptom } & Baseline & Intake 1 & Intake 2 & Follow-up \\
\hline Painful effort during defecation & $1.67(1.29)$ & $1.45(1.14)^{\mathrm{a}}$ & $1.15(0.97)^{\mathrm{a}}$ & $1.16(0.88)^{\mathrm{a}}$ \\
Feeling of incomplete evacuation & $2.27(1.03)$ & $2.01(1.31)^{\mathrm{a}}$ & $1.61(1.28)^{\mathrm{a}}$ & $1.53(1.32)^{\mathrm{a}}$ \\
Straining during defecation & $2.38(0.83)$ & $2.00(0.67)^{\mathrm{a}}$ & $1.75(1.19)^{\mathrm{a}}$ & $1.71(1.17)^{\mathrm{a}}$ \\
Minutes spent in lavatory per attempt & $1.90(1.06)$ & $1.68(0.90)$ & $1.68(0.80)^{\mathrm{a}}$ & $1.66(0.94)^{\mathrm{a}}$ \\
Unsuccessful defecation attempt & $1.13(0.40)$ & $1.08(0.43)$ & $1.09(0.28)$ & $1.00(0.14)^{\mathrm{a}}$ \\
Abdominal discomfort & $1.97(1.03)$ & $1.92(1.10)$ & $1.60(1.03)^{\mathrm{a}}$ & $1.30(1.08)^{\mathrm{a}}$ \\
Defecation frequency (times/wk) & $4.00(2.50)$ & $4.50(2.50)$ & $5.00(2.50)^{\mathrm{a}}$ & $5.25(2.63)^{\mathrm{a}}$ \\
Stool consistency & $3.18(1.02)$ & $3.44(1.41)^{\mathrm{a}}$ & $3.55(1.02)^{\mathrm{a}}$ & $3.19(1.21)$ \\
\hline
\end{tabular}

${ }^{\mathrm{a}} \mathrm{P}<0.05$, vs baseline.

Data are presented as mean $\pm \mathrm{SD}$.

Intake 1, 2-week Lactobacillus casei strain Shirota (LcS) intake; intake 2, 4-week LcS intake; follow-up, 2-week following LcS intake.

Data are presented as median (interquartile range). 


\section{Effect of Lactobacillus casei Strain Shirota on Stool Consistency: Subanalysis Based on Bristol Stool Form Scale}

After both 2 and 4 weeks of LcS intervention, the stool consistencies were noticeably improved (Table 2). The effect of LcS on stool consistency varied among the different groups (Table 3). In the HS group, LcS supplementation significantly increased the BSFS score (from 2.50 to 3.04 after 4 weeks of intervention). However, in the SS group, LcS intake reduced the BSFS score (from 4.46 to 4.14 after 4 weeks of intervention). However, the BSFS score did not change substantially with LcS intervention in the NS group. These results suggested that LcS could effectively improve the stool consistency of constipated patients with hard stools.

Table 3. Effects of Lactobacillus casei Strain Shirota on Stool Consistency

\begin{tabular}{ccccc}
\hline Group & Baseline & Intake 1 & Intake 2 & Follow-up \\
\hline HS & $2.50(1.13)$ & $2.75(0.84)^{\mathrm{a}}$ & $3.04(1.30)^{\mathrm{a}}$ & $3.00(1.43)^{\mathrm{a}}$ \\
NS & $3.25(0.39)^{\mathrm{b}}$ & $3.63(1.00)$ & $3.63(0.82)$ & $3.25(1.00)$ \\
SS & $4.46(0.39)^{\mathrm{b}, \mathrm{c}}$ & $4.50(1.10)$ & $4.14(1.40)^{\mathrm{a}}$ & $4.02(1.76)^{\mathrm{a}}$ \\
\hline
\end{tabular}

${ }^{\mathrm{a}} P<0.05$, vs baseline.

${ }^{\mathrm{b}} P<0.05$, vs HS.

${ }^{\mathrm{c}} P<0.05$, vs NS.

HS, hard stool; NS, normal stool; SS, soft stool; intake 1, 2-week Lactobacillus casei strain Shirota (LcS) intake; intake 2, 4-week LcS intake; follow-up, 2-week following LcS intake.

Data are presented as median (interquartile range).

Table 4. Effect of Lactobacillus casei Strain Shirota on Constipation Symptoms of Each Group

\begin{tabular}{|c|c|c|c|c|c|}
\hline Symptom & Group & Baseline & Intake 1 & Intake 2 & Follow-up \\
\hline \multirow[t]{3}{*}{ Painful effort during defecation } & HS & $1.75(0.99)$ & $1.14(0.88)^{\mathrm{a}}$ & $1.00(0.57)^{\mathrm{a}}$ & $1.00(0.46)^{\mathrm{a}}$ \\
\hline & NS & $1.69(1.29)$ & $1.71(1.14)^{\mathrm{a}}$ & $1.25(1.14)^{\mathrm{a}}$ & $1.29(0.9)^{\mathrm{a}}$ \\
\hline & SS & $1.44(1.30)$ & $1.86(1.35)$ & $1.66(1.63)$ & $1.69(1.20)$ \\
\hline \multirow[t]{3}{*}{ Feeling of incomplete evacuation } & HS & $2.50(1.09)$ & $2.00(0.99)^{\mathrm{a}}$ & $1.46(0.73)^{\mathrm{a}}$ & $1.22(1.12)^{\mathrm{a}}$ \\
\hline & NS & $2.20(0.84)$ & $1.86(1.33)$ & $1.50(1.72)^{\mathrm{c}}$ & $1.36(1.50)^{\mathrm{a}}$ \\
\hline & SS & $2.37(1.31)$ & $2.55(1.21)$ & $2.23(1.04)$ & $1.73(0.75)$ \\
\hline \multirow[t]{3}{*}{ Straining during defecation } & HS & $2.76(1.00)$ & $2.00(0.62)^{\mathrm{a}}$ & $1.56(0.79)^{\mathrm{a}}$ & $1.56(0.92)^{\mathrm{a}}$ \\
\hline & NS & $2.25(0.54)^{\mathrm{b}}$ & $2.00(1.04)$ & $2.00(1.22)^{\mathrm{a}}$ & $1.90(1.10)^{\mathrm{a}}$ \\
\hline & SS & $1.97(0.9)^{\mathrm{b}, \mathrm{c}}$ & $2.00(0.59)$ & $1.84(1.32)$ & $1.52(1.20)^{\mathrm{a}}$ \\
\hline \multirow[t]{3}{*}{ Minutes spent in lavatory per attempt } & HS & $1.92(1.17)$ & $1.57(0.81)$ & $1.75(0.71)^{\mathrm{a}}$ & $1.79(0.79)^{\mathrm{a}}$ \\
\hline & NS & $1.91(1.13)$ & $2.00(0.69)$ & $1.80(0.92)$ & $1.92(1.14)$ \\
\hline & SS & $1.44(1.00)$ & $1.25(0.93)$ & $1.25(0.95)$ & $1.22(0.75)$ \\
\hline \multirow[t]{3}{*}{ Unsuccessful defecation attempt } & HS & $1.35(0.86)$ & $1.08(0.43)$ & $1.04(0.21)^{\mathrm{a}}$ & $1.00(0.14)^{\mathrm{a}}$ \\
\hline & NS & $1.13(0.33)$ & $1.09(0.50)^{\mathrm{a}}$ & $1.06(0.28)^{\mathrm{a}}$ & $1.08(0.15)^{\mathrm{a}}$ \\
\hline & SS & $1.00(0.22)$ & $1.07(0.40)$ & $1.21(0.5)^{\mathrm{a}}$ & $1.00(0.08)$ \\
\hline \multirow{3}{*}{ Abdominal discomfort } & HS & $1.90(1.37)$ & $1.86(1.10)$ & $1.57(0.84)^{\mathrm{a}}$ & $1.31(0.74)^{\mathrm{a}}$ \\
\hline & NS & $2.00(0.92)$ & $1.74(0.97)^{\mathrm{a}}$ & $1.29(1.19)^{\mathrm{a}}$ & $1.16(1.06)^{\mathrm{a}}$ \\
\hline & SS & $2.29(1.56)$ & $2.42(1.05)$ & $2.39(1.34)$ & $1.93(1.64)$ \\
\hline \multirow[t]{3}{*}{ Defecation frequency } & HS & $4.50(3.50)$ & $4.50(3.00)$ & $5.00(3.00)^{\mathrm{a}}$ & $5.00(3.00)^{\mathrm{a}}$ \\
\hline & NS & $4.00(2.00)$ & $4.50(2.50)$ & $5.00(2.00)^{\mathrm{a}}$ & $5.50(3.00)^{\mathrm{a}}$ \\
\hline & SS & $4.25(1.25)$ & $3.75(2.38)$ & $5.25(1.88)^{\mathrm{a}}$ & $5.25(2.13)$ \\
\hline
\end{tabular}

${ }^{\mathrm{a}} \mathrm{P}<0.05$, vs baseline.

${ }^{\mathrm{b}} \mathrm{P}<0.05$, vs HS.

${ }^{\mathrm{c}} P<0.05$, vs NS.

HS, hard stool; NS, normal stool; SS, soft stool; intake 1, 2-week Lactobacillus casei strain Shirota (LcS) intake; intake 2, 4-week LcS intake; follow-up, 2-week following LcS intake.

Data are presented as median (interquartile range). 


\section{Effect of Lactobacillus casei Strain Shirota on Other Constipation-related Symptoms: Subanalysis Based on Bristol Stool Form Scale}

LcS supplementation was more effective at treating constipation-related symptoms in the HS group than in the NS and SS groups (Table 4). Four weeks of $\mathrm{LcS}$ supplementation significantly alleviated the minutes spent in the lavatory per attempt only in the HS group. In addition, the symptoms of pain, feeling of incomplete evacuation, straining during defecation, unsuccessful defecation and abdominal discomfort were alleviated in the HS and NS groups but not the SS group. The defecation frequency improved in all the groups.

\section{Effect of Lactobacillus casei Strain Shirota on Stool Short Chain Fatty Acids}

The baseline SCFA concentrations differed among the 3 groups. The SS group had the highest levels of all the SCFAs, followed by the NS group and then the HS group (Table 5), and this order was consistent with that of the BSFS scores. Therefore, we further analysed the relationship between stool SCFAs and the BSFS score. Significant correlation was observed between SCFA levels and BSFS scores (acetate $\left[\mathrm{r}=0.41, \mathrm{q}=2.63 \times 10^{-3}\right]$, propionate $\left[\mathrm{r}=0.37, \mathrm{q}=4.37 \times 10^{-3}\right]$, and butyrate $[\mathrm{r}=0.34, \mathrm{q}=$ $\left.6.44 \times 10^{-3} \mathrm{]}\right)$.

LcS intake had little influence on stool SCFA levels in the 62 constipated subjects. However, the effect of $\mathrm{LcS}$ ingestion varied among the 3 groups. As shown in Table 5, consumption of the LcSfermented dairy beverage for 2 weeks could enhance stool acetate, propionate, and butyrate levels by $35.1 \%, 36.8 \%$, and $39.7 \%$, respectively, in the HS group $(P=0.052,0.033$, and 0.031 , respectively). In contrast, the SCFA levels decreased (28.3\%, 19.3\%, and $18.6 \%$ for acetate, propionate and butyrate, respectively) in the SS group (not significant). No significant change was observed during LcS intervention in the NS group (except for a significant decrease in acetate levels at the intake 1 visit; the acetate level reverted to the baseline level at the intake 2 visit). Therefore, the results indicated that the effect of $\mathrm{LcS}$ intervention on stool SCFA levels varied according to original stool consistency.

\section{Effect of Lactobacillus casei Strain Shirota on the Gut Microbiota}

We compared the baseline gut microbiota from each of the 3 groups with that observed during $\mathrm{LcS}$ supplementation. Baseline stool samples and those from the intake 1 visit (as representatives of the intake 1 and 2 visits) were subjected to next-generation sequencing of the bacterial 16S rRNA V3-V4 region on an Illumina MiSeq platform.

A total of 5694382 filtered high-quality sequence reads were obtained (with a mean of 45922 reads/sample and an average length of 432 bp), clustered and binned into OTUs at a 97\% similarity level with Usearch. Samples were rarefied to a depth of 17764 per sample. A total of 509 OTUs (belonging to 117 genera) together with their representative sequences were obtained. The rarefaction

Table 5. Effects of Lactobacillus casei Strain Shirota on Short Chain Fatty Acids

\begin{tabular}{|c|c|c|c|c|c|}
\hline SCFA & Group & Baseline & Intake 1 & Intake 2 & Follow-up \\
\hline \multirow[t]{4}{*}{ Acetate } & HS & $21.49(24.20)$ & $29.37(29.67)^{\mathrm{a}}$ & $22.94(35.25)^{\mathrm{a}}$ & $14.25(25.24)$ \\
\hline & NS & $32.03(28.18)$ & $18.70(18.77)^{\mathrm{b}}$ & $27.12(23.65)$ & $19.51(28.78)^{a}$ \\
\hline & SS & $44.54(11.64)^{c}$ & $29.61(33.07)$ & $34.98(20.07)$ & $21.73(20.91)^{b}$ \\
\hline & Total & $30.65(29.84)$ & $24.32(26.31)$ & $30.24(24.96)$ & $18.24(25.86)^{b}$ \\
\hline \multirow[t]{4}{*}{ Propionate } & HS & $12.30(10.98)$ & $14.92(10.49)^{\mathrm{b}}$ & $13.32(8.33)$ & $10.93(12.81)$ \\
\hline & NS & $15.17(13.37)$ & $13.91(7.98)$ & $14.06(13.22)$ & $13.63(9.81)^{b}$ \\
\hline & $\mathrm{SS}$ & $18.67(18.94)^{\mathrm{c}}$ & $18.30(16.69)$ & $19.46(14.33)$ & $14.41(8.59)^{b}$ \\
\hline & Total & $15.42(12.26)$ & $14.81(9.21)$ & $14.15(10.85)$ & $11.39(10.04)^{b}$ \\
\hline \multirow[t]{4}{*}{ Butyrate } & $\mathrm{HS}$ & $8.37(12.28)$ & $16.18(9.06)^{b}$ & $11.22(12.91)$ & $9.73(15.46)$ \\
\hline & NS & $14.64(10.60)$ & $13.53(12.67)$ & $13.33(14.10)$ & $10.15(12.22)$ \\
\hline & $\mathrm{SS}$ & $17.41(22.75)^{c}$ & $14.43(20.77)$ & $17.13(15.02)$ & $9.73(13.72)^{b}$ \\
\hline & Total & $14.34(12.33)$ & $14.43(11.11)$ & $12.91(13.89)$ & $9.97(13.64)$ \\
\hline
\end{tabular}

${ }^{\mathrm{a}} P<0.1$, vs baseline.

${ }^{\mathrm{b}} P<0.05$, vs baseline.

${ }^{\mathrm{c}} P<0.05$, vs $\mathrm{HS}$.

HS, hard stool; NS, normal stool; SS, soft stool; intake 1, 2-week LcS intake; intake 2, 4-week LcS intake; follow-up, 2-week following LcS intake.

Data are presented as median (interquartile range). 
curves and Shannon diversity indices of all the samples were stable, suggesting that rare new phylotypes would appear on further sequencing and that most of the diversity had already been covered. Then, $99.0 \%$ of the sequences were assigned into 11 different phyla with a RDP Classifier. Firmicutes, Bacteroidetes, Actinobacteria, and Proteobacteria were the most abundant phyla, with relative abundances of $66.9 \%, 22.6 \%, 8.9 \%$, and $1.2 \%$, respectively.

Adonis, based on weighted UniFrac distances, was applied to compare the overall gut microbiota composition among each group and between baseline and post-LcS-intake visits. LcS intervention significantly altered the gut microbiota $(P=0.031)$. The baseline gut microbiota also differed among each group $(P=0.086)$, although not significantly. PCoA based on weighted UniFrac dissimilarity was applied to provide an overview of the gut microbiota in the 3 groups at the baseline and intake 1 periods (Fig. 2A). The first 2 principal components (PCs) collectively accounted for $72.8 \%$ of the total variation. $\mathrm{PC} 1$ and $\mathrm{PC} 2$ constituted $47.5 \%$ and $25.4 \%$ of the total variation, respectively. Plotted PCoA scores suggested that there were no obvious differences in the gut microbiota. To compare variations between and within periods, ANOSIM based on weighted UniFrac distances was used, and the data showed that the dissimilarity between periods and within periods were even $(\mathrm{R}$ $=0.015, P=0.077$ ). Therefore, variations in the gut microbiota among individuals masked the changes in the gut microbiota caused by LcS. Thus, the PCoA plot did not separate clearly among periods, even if $\mathrm{PCoA}$ was performed in an individual group (Fig. 2BD).

Genera belonging to Lachnospiraceae and Ruminococcaceae varied among the 3 groups. The relative abundances of the Lachnospiraceae FCS020 group was highest in the $\mathrm{SS}$ group $(P=$
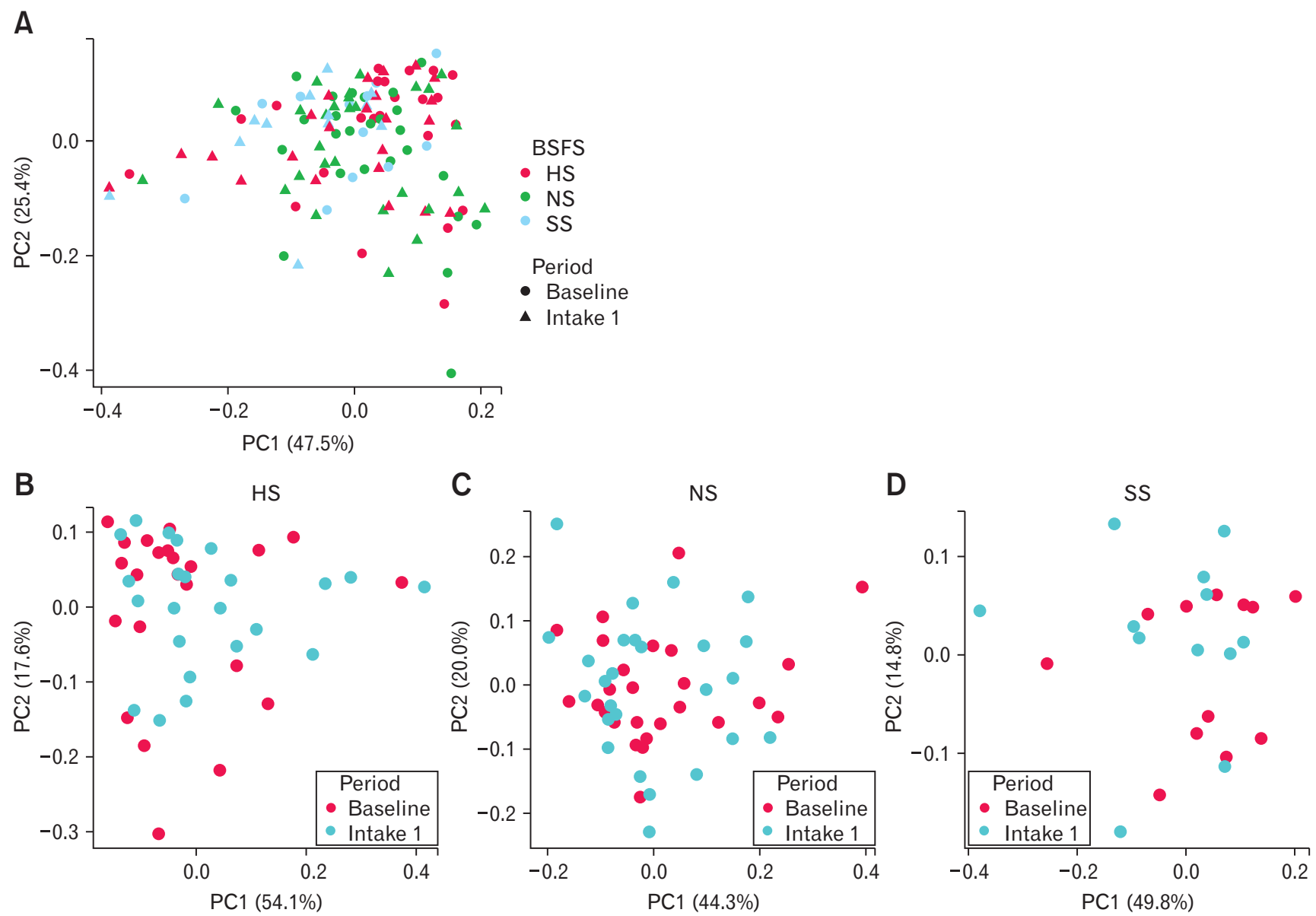

Figure 2. Response of the gut microbiota to Lactobacillus casei strain Shirota $(\mathrm{LcS})$ intervention. Plots were based on principal coordinate analyses with weighted UniFrac dissimilarities. The gut microbiota of all the subjects (A), the hard stool (HS) group (B), the normal stool (NS) group (C), and the soft stool (SS) group (D) at the baseline period and 2-week LcS intake (intake 1) visit was included in this analysis. BSFS, Bristol Stool Form Scale; PC, principal component. 
A

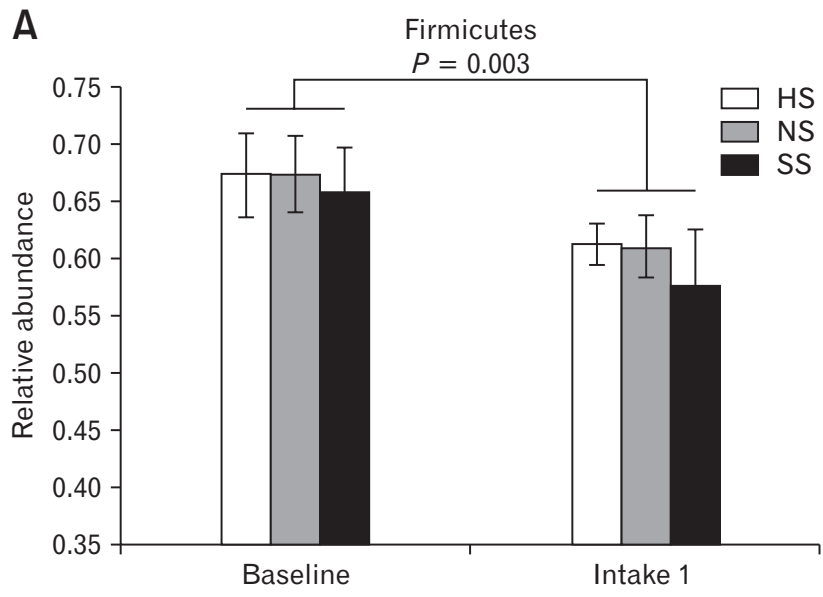

C

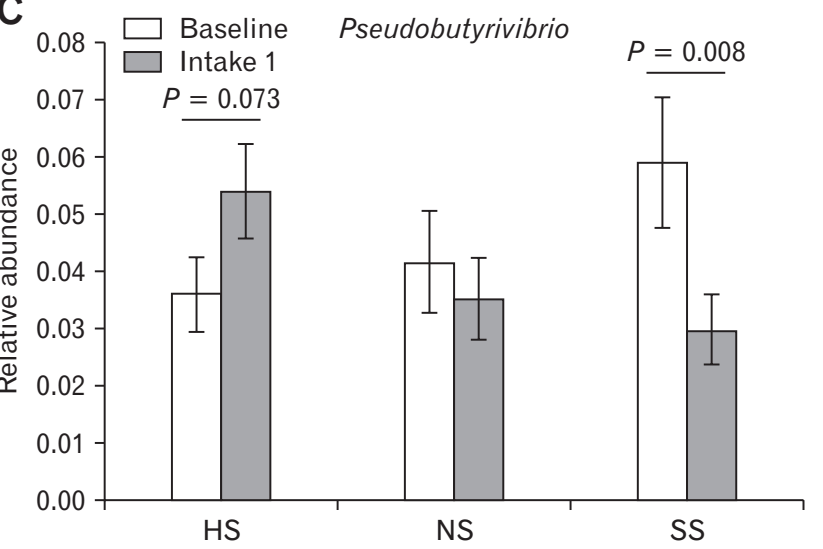

B

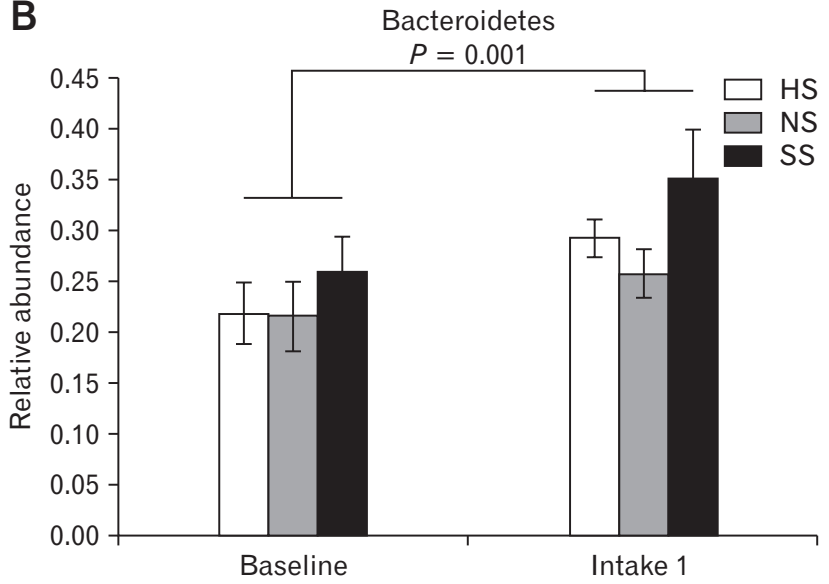

D

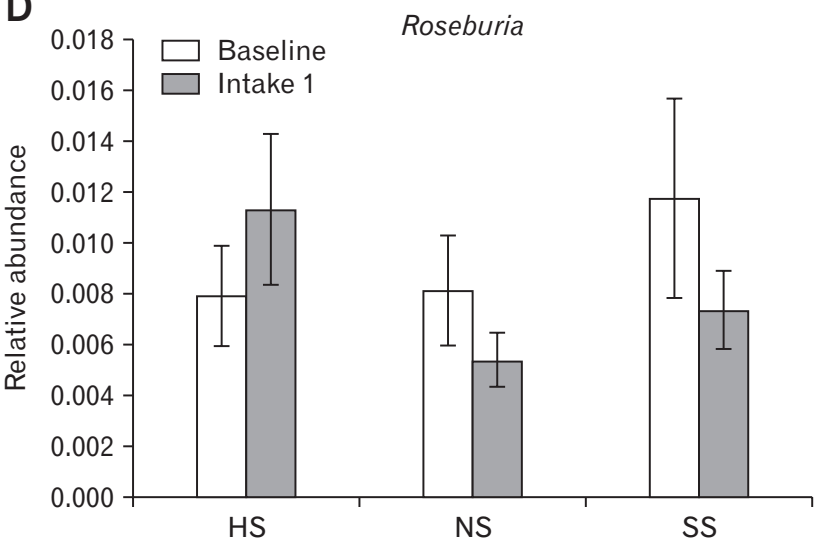

E

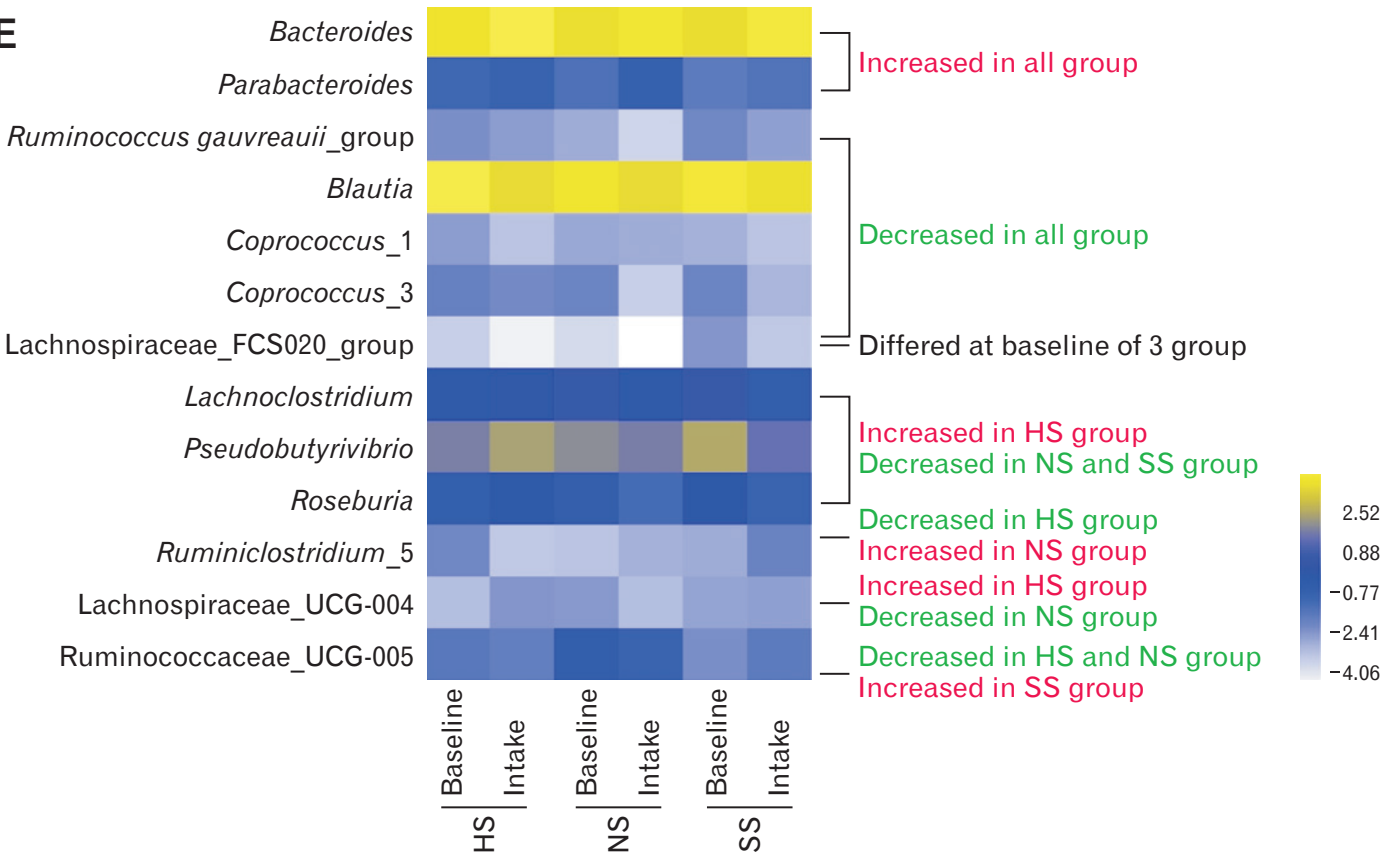

Figure 3. Relative abundances of bacterial taxa affected by Lactobacillus casei strain Shirota (LcS) intervention. The phyla (A) Firmicutes and (B) Bacteroidetes and genera (C) Pseudobutyrivibrio, (D) Roseburia, and (E) others. P-values were derived by repeated measures ANOVA. HS, hard stool; NS, normal stool; SS, soft stool; intake 1, 2-week LcS intake. 
0.004), while Ruminococcaceae UCG-005 was most abundant in the NS group $(P=0.088)$ (Fig. 3E).

Although potential modification in the overall gut microbiota may be masked by intersubject variability, LcS intervention modulated the relative abundances of specific microbes. At the phylum level, LcS intake significantly increased the relative abundance of Bacteroidetes and decreased the relative abundance of Firmicutes (Fig. 3A and 3B). Meanwhile, LcS intake significantly decreased the relative abundance of genera belonging to Lachnospiraceae, such as Blautia, Coprococcus_1, Coprococcus_3, the Lachnospiraceae FCS020 group and the Ruminococcus gauvreauii group in all 3 groups but increased the relative abundances of Bacteroides and Parabacteroides (both belonging to Bacteroidetes) (Fig. 3E). Repeated measures ANOVA was used to elucidate the interaction of the BSFS group and $\mathrm{LcS}$ intake in terms of the microbiota. The BSFS level at the baseline period visibly interacted with $\mathrm{LcS}$ intervention in terms of the relative abundances of Lachnoclostridium, Lachnospiraceae UCG-004, Pseudobutyrivibrio and Ruminiclostridium 5 (Fig. 3E). In addition, the baseline BSFS level and LcS intake also had an interactive effect on Roseburia, but this effect was not significant $(P=0.070$; Fig. 3D). Therefore, the baseline BSFS level may influence the effect of $\mathrm{LcS}$ intake on the microbiota. Pseudobutyrivibrio and Roseburia were formerly reported as butyrate producers, and $\mathrm{LcS}$ intake induced an increase in the abundance of these 2 genera by $50.5 \%$ and $43.0 \%$, respectively, in the HS group but suppressed the abundances by $49.7 \%$ and $41.9 \%$, respectively, in the SS group (Fig. 3C and 3D), which was consistent with the change in butyrate levels.

\section{Discussion}

In this study, the effectiveness of $\mathrm{LcS}$ in the treatment of constipation was evaluated. Further, stool consistency assessed with the BSFS was used to classify the subjects into 3 groups (the HS, NS, and SS groups). Differences between baseline data and outcomes after LcS intervention for constipation-related symptoms, stool SCFA levels and gut microbial composition were observed among the 3 groups with different baseline stool consistencies.

In general, LcS supplementation significantly relived constipation symptoms. After receiving LcS supplementation, patients defecated with increased frequency and ease. In addition, the stool consistency improved. Consistent with a study performed in Germany, these results suggested the effectiveness of $\mathrm{LcS}$ in the alleviation of constipation. $^{23}$

Stool consistency is one of the most important parameters asso- ciated with constipation, as it can serve as a reliable proxy for colonic transit rate, and abnormal colonic transit is considered a primary cause of constipation. ${ }^{16,24,25}$ Stool consistency was also associated with the species richness, enterotypes and community composition of the gut microbiota in stool samples of 53 healthy women and in LifeLines-DEEP, a large population-based cohort. ${ }^{26,27}$ We also noted differences in the gut microbiota (mainly Lachnospiraceae bacteria) among the 3 groups with different baseline stool consistencies.

Although the etiology of constipation remains not fully understood, growing evidence indicates that alterations in the gut microbiota may contribute to constipation and related symptoms. The gut microbiota of constipated patients was reported to differ from that of healthy adults and children. ${ }^{28-30}$ Additionally, dysbiosis of the gut microbiota could influence gastrointestinal motility and eventually lead to constipation. ${ }^{31}$ Studies of gnotobiotic and germ-free animals confirmed that the gut microbiota could influence gastrointestinal motility in a species-specific manner. ${ }^{31,32}$ Colonization of the conventional gut microbiota of germ-free rats with Lactobacillus acidophilus and Bifidobacterium bifidum accelerated small-intestinal transit, but Micrococcus luteus and Escherichia coli had the opposite effect. ${ }^{32}$ Thus, we sought to understand how the gut microbiota guides optimal management of constipation with multiple symptoms and diverse aetiology.

The effects of LcS intervention on the gut microbiota depended on the baseline stool consistency (Fig. 3). The abundances of Pseudobutyrivibrio and Roseburia, which are butyrate-producing bacteria, increased in the HS group but decreased in the SS group. The abundances of these 2 genera did not change in the NS group. In previous studies, an identical intervention had diverse, even opposite, effects on the gut microbiota. Venkataraman et $\mathrm{al}^{33}$ reported that supplementation with resistant starch increased the abundances of resistant-starch-degrading intestinal microbes, leading to elevated butyrate content in individuals with high initial stool butyrate levels, whereas no effect was observed in subjects with low initial stool butyrate levels. Ferrario et $\mathrm{al}^{21}$ showed that Lactobacillus paracasei DG intervention could reduce butyrate content and decrease the abundances of genera belonging to Clostridiales in subjects with high stool butyrate content. In contrast, the same bacterial supplementation increased the butyrate levels and Bacteroidales abundances in subjects with low stool butyrate content, but with a decrease in $R u$ minococcus abundance. ${ }^{21}$ Kovatcheva-Datchary et $\mathrm{al}^{34}$ showed that subjects enriched with Prevotella exhibited improved glucose metabolism during the consumption of barley kernel-based bread, but subjects with low abundances of Prevotella did not respond to this diet. Thus, the initial characteristics of the intestinal environment 
and/or microbial ecosystem are important for effective probiotic or prebiotic intervention.

The changes in the levels of butyrate-producing bacteria resulted in varied responses to LcS intervention based on stool butyrate levels. LcS intervention increased the stool butyrate levels in HS but not in NS. LcS reduced the butyrate levels in SS (Table 5). Studies suggest that the gut microbiota influences gut motility via production of SCFAs. Direct infusion of SCFAs into the proximal colons of rats significantly accelerated colonic transit. ${ }^{35,36}$ SCFAs, especially butyrate, can serve as an energy source for enterocytes and regulate gut motility via the release of 5-hydroxytryptamine and polypeptide YY by enterocytes or altered cholinergic phenotypes. ${ }^{35-37}$ The data from the present study show that the baseline stool SCFA levels were highest in the SS group and lowest in the HS group (Table 5). A positive correlation was also observed between each SCFA and the BSFS scores, which indicated an association between SCFAs and gut motility in constipation. The results of Oufir's study were consistent with our findings. ${ }^{38}$ They directly measured colonic transit time and SCFA content in healthy subjects and observed an inverse correlation between the two parameters. ${ }^{38}$

Varying effects of LcS intervention on stool consistency were observed among the 3 groups (Table 4). In the HS group, softer stool was observed during $\mathrm{LcS}$ supplementation than during the baseline period, indicating that intake of $\mathrm{LcS}$ significantly accelerated colonic transit in the HS group. It was reported that $\mathrm{LcS}$ supplementation shortened the colonic transit time in slow-transit constipation, hence reducing the occurrence of hard or lumpy stools in healthy and constipated subjects. ${ }^{13,14,39}$ Increased stool SCFA concentrations and abundances of butyrate-producing bacteria were observed in the HS group, whose stool was significantly softened by $\mathrm{LcS}$ intervention. However, stool consistency remained unchanged in the NS group, and the stool became harder in the SS group during LcS supplementation. This finding is consistent with a previous study, in which LcS accelerated colonic transit in slow-transit constipation and had only a modest effect or no effect on constipation with normal colonic transit. $^{15,23,39}$ Decreased SCFA concentrations and abundances of butyrate-producing bacteria were also observed in the SS group. We believe that the normalization of gastrointestinal motility to the levels of SCFAs, especially butyric acid, is one of the factors that contributes to the improvement of stool consistency by $\mathrm{LcS}$.

The limitation of this study was that we did not employ the validated scales for the evaluation of constipation-related symptoms, such as the Patient Assessment of Constipation Symptoms or the Patient Assessment of Constipation Quality of Life questionnaire. ${ }^{40,41}$ These validated assessment tools need to be included in future studies to confirm our findings.

In conclusion, we divided the constipated patients based on baseline stool consistency, and $\mathrm{LcS}$ supplementation improved the constipation symptoms in all the subjects. Subanalysis based on stool consistency revealed that $\mathrm{LcS}$ balanced the stool consistencysoftened the hard stool and hardened the soft stool—probably by modulating the composition of and SCFA production by the gut microbiota.

Financial support: This study was supported by Yakult Honsha Co, Ltd.

\section{Conflicts of interest: None.}

Author contributions: Shanbin Chen and Yangwenshan $\mathrm{Ou}$ conducted the study and drafted the initial manuscript; Shanbin Chen, Liang Zhao, and Fazheng Ren designed the study; Yang $\mathrm{Li}$ and Zhenxing Qiao contributed to data collection and interpretation; Liang Zhao, Yanling Hao, and Fazheng Ren critically reviewed the manuscript; and all authors approved the final version of the manuscript and agreed to be accountable for all aspects of the work by ensuring that questions related to the accuracy or integrity of any part of the work are appropriately investigated and resolved.

\section{References}

1. Chu H, Zhong L, Li H, Zhang X, Zhang J, Hou X. Epidemiology characteristics of constipation for general population, pediatric population, and elderly population in china. Gastroenterol Res Pract 2014;2014:532734.

2. Sun SX, Dibonaventura M, Purayidathil FW, Wagner JS, Dabbous O, Mody R. Impact of chronic constipation on health-related quality of life, work productivity, and healthcare resource use: an analysis of the national health and wellness survey. Dig Dis Sci 2011;56:2688-2695.

3. Pekmezaris R, Aversa L, Wolf-Klein G, Cedarbaum J, Reid-Durant M. The cost of chronic constipation. J Am Med Dir Assoc 2002;3:224-228.

4. Ashraf W, Park F, Lof J, Quigley EM. An examination of the reliability of reported stool frequency in the diagnosis of idiopathic constipation. Am J Gastroenterol 1996;91:26-32.

5. Sandler RS, Drossman DA. Bowel habits in young adults not seeking health care. Dig Dis Sci 1987;32:841-845.

6. Shih DQ, Kwan LY. All roads lead to Rome: update on Rome III criteria and new treatment options. Gastroenterol Rep 2007;1:56-65.

7. Glia A, Lindberg G. Quality of life in patients with different types of functional constipation. Scand J Gastroenterol 1997;32:1083-1089.

8. Zhao Y, Yu YB. Intestinal microbiota and chronic constipation. Springerplus 2016;5:1130.

9. Chmielewska A, Szajewska H. Systematic review of randomised controlled trials: probiotics for functional constipation. World J Gastroenterol 
2010;16:69-75.

10. Dimidi E, Christodoulides S, Fragkos KC, Scott SM, Whelan K. The effect of probiotics on functional constipation in adults: a systematic review and meta-analysis of randomized controlled trials. Am J Clin Nutr 2014;100:1075-1084.

11. Del Piano M, Carmagnola S, Anderloni A, et al. The use of probiotics in healthy volunteers with evacuation disorders and hard stools: a double-blind, randomized, placebo-controlled study. J Clin Gastroenterol 2010;44(suppl 1):S30-S34.

12. Takii H, Nishijima T, Takami K, et al. Effects of fermented milk containing Bifidobacterium animalis subsp. lactis GCL2505 on improvement of defecation, fecal properties, and intestinal microflora in healthy subjects with mild constipation. Jpn Pharmacol Ther 2012;40:457-465.

13. Sakai T, Makino H, Ishikawa E, Oishi K, Kushiro A. Fermented milk containing Lactobacillus casei strain Shirota reduces incidence of hard or lumpy stools in healthy population. Int J Food Sci Nutr 2011;62:423430 .

14. Tilley L, Keppens K, Kushiro A, et al. A probiotic fermented milk drink containing Lactobacillus Casei strain Shirota improves stool consistency of subjects with hard stools. Int J Probiotics Prebiotics 2014;9:23-29.

15. Mazlyn MM, Nagarajah LH, Fatimah A, Norimah AK, Goh KL. Effects of a probiotic fermented milk on functional constipation: a randomized, double-blind, placebo-controlled study. J Gastroenterol Hepatol 2013;28:1141-1147.

16. Lewis SJ, Heaton KW. Stool form scale as a useful guide to intestinal transit time. Scand J Gastroenterol 1997;32:920-924.

17. Mínguez Pérez M, Benages Martínez A. The Bristol scale: a useful system to assess stool form? Rev Esp Enfem Dig 2009;101:305-311.

18. Goossens D, Jonkers D, Russel M, Stobberingh E, Van Den Bogaard A, StockbrUgger R. The effect of Lactobacillus plantarum 299v on the bacterial composition and metabolic activity in faeces of healthy volunteers: a placebo-controlled study on the onset and duration of effects. Aliment Pharmacol Ther 2003;18:495-505.

19. Via LE, Falkinham III JO. Comparison of methods for isolation of mycobacterium-avium complex dna for use in pcr and rapd fingerprinting. $\mathrm{J}$ Microbiol Methods 1995;21:151-161.

20. Amato KR, Yeoman CJ, Kent A, et al. Habitat degradation impacts black howler monkey (Alouatta pigra) gastrointestinal microbiomes. ISME J 2013;7:1344-1353.

21. Ferrario C, Taverniti V, Milani C, et al. Modulation of fecal Clostridiales bacteria and butyrate by probiotic intervention with Lactobacillus paracasei DG varies among healthy adults. J Nutr 2014;144:1787-1796.

22. Lozupone CA, Hamady M, Kelley ST, Knight R. Quantitative and qualitative beta diversity measures lead to different insights into factors that structure microbial communities. Appl Environ Microbiol 2007;73:1576-1585.

23. Koebnick C, Wagner I, Leitzmann P, Stern U, Zunft H. Probiotic beverage containing Lactobacillus casei Shirota improves gastrointestinal symptoms in patients with chronic constipation. Can J Gastroenterol 2003;17:655-659.

24. Russo M, Martinelli M, Sciorio E, et al. Stool consistency, but not frequency, correlates with total gastrointestinal transit time in children. J
Pediatr 2013;162:1188-1192.

25. Wang HL. Understanding the pathogenesis of slow-transit constipation: one step forward. Dig Dis Sci 2015;60:2216-2218.

26. Vandeputte D, Falony G, Vieira-Silva S, Tito RY, Joossens M, Raes J. Stool consistency is strongly associated with gut microbiota richness and composition, enterotypes and bacterial growth rates. Gut 2016;65:57-62.

27. Tigchelaar EF, Bonder MJ, Jankipersadsing SA, Fu J, Wijmenga C, Zhernakova A. Gut microbiota composition associated with stool consistency. Gut 2016;65:540-542.

28. Khalif IL, Quigley EM, Konovitch EA, Maximova ID. Alterations in the colonic flora and intestinal permeability and evidence of immune activation in chronic constipation. Dig Liver Dis 2005;37:838-849.

29. Zoppi G, Cinquetti M, Luciano A, Benini A, Muner A, Bertazzoni Minelli $\mathrm{E}$. The intestinal ecosystem in chronic functional constipation. Acta Paediatr 1998;87:836-841

30. de Moraes JG, Motta ME, Beltrão MF, Salviano TL, da Silva GA. Fecal microbiota and diet of children with chronic constipation. Int J Pediatr 2016;2016:6787269.

31. Abrams GD. Microbial effects on mucosal structure and function. Am J Clin Nutr 1977;30:1880-1886.

32. Husebye E, Hellström PM, Sundler F, Chen J, Midtvedt T. Influence of microbial species on small intestinal myoelectric activity and transit in germ-free rats. Am J Physiol Gastrointest Liver Physiol 2001;280:G368G380.

33. Venkataraman A, Sieber JR, Schmidt AW, Waldron C, Theis KR, Schmidt TM. Variable responses of human microbiomes to dietary supplementation with resistant starch. Microbiome 2016;4:33.

34. Kovatcheva-Datchary P, Nilsson A, Akrami R, et al. Dietary fiberinduced improvement in glucose metabolism is associated with increased abundance of prevotella. Cell Metab 2015;22:971-982.

35. Cherbut C, Ferrier L, Rozé C, et al. Short-chain fatty acids modify colonic motility through nerves and polypeptide YY release in the rat. Am J Physiol 1998;275(6 Pt 1):G1415-G1422.

36. Fukumoto S, Tatewaki M, Yamada T, et al. Short-chain fatty acids stimulate colonic transit via intraluminal 5-HT release in rats. Am J Physiol Regul Integr Comp Physiol 2003;284:R1269-R1276.

37. Soret R, Chevalier J, De Coppet $\mathrm{P}$, et al. Short-chain fatty acids regulate the enteric neurons and control gastrointestinal motility in rats. Gastroenterology 2010;138:1772-1782.

38. Oufir LE, Barry JL, Flourié B, et al. Relationships between transit time in man and in vitro fermentation of dietary fiber by fecal bacteria. Eur J Clin Nutr 2000;54:603-609.

39. Krammer HJ, von Seggern H, Schaumburg J, Neumer F. Effect of Lactobacillus casei Shirota on colonic transit time in patients with chronic constipation. Coloproctol 2011;33:109-113.

40. Frank L, Kleinman L, Farup C, Taylor L, Miner P Jr. Psychometric validation of a constipation symptom assessment questionnaire. Scand $\mathrm{J}$ Gastroenterol 1999;34:870-877.

41. Marquis P, De La Loge C, Dubois D, McDermott A, Chassany O. Development and validation of the patient assessment of constipation quality of life questionnaire. Scand J Gastroenterol 2005;40:540-551. 